University of Texas at El Paso

ScholarWorks@UTEP

$2-2018$

\title{
Optimization of Quadratic Forms and t-norm Forms on Interval Domain and Computational Complexity
}

\author{
Milan Hladik \\ Charles University, Faculty of Mathematics and Physics, Department of Applied Mathematics, Prague, \\ milan.hladik@matfyz.cz \\ Michal Čerńy \\ University of Economics, Prague, cernym@vse.cz \\ Vladik Kreinovich \\ The University of Texas at El Paso, vladik@utep.edu
}

Follow this and additional works at: https://scholarworks.utep.edu/cs_techrep

Part of the Computer Sciences Commons

Comments:

Technical Report: UTEP-CS-18-14

To appear in Proceedings of the World Conference on Soft Computing, Baku, Azerbaijan, May 29-31, 2018.

\section{Recommended Citation}

Hladik, Milan; Čerńy, Michal; and Kreinovich, Vladik, "Optimization of Quadratic Forms and t-norm Forms on Interval Domain and Computational Complexity" (2018). Departmental Technical Reports (CS). 1204. https://scholarworks.utep.edu/cs_techrep/1204

This Article is brought to you for free and open access by the Computer Science at ScholarWorks@UTEP. It has been accepted for inclusion in Departmental Technical Reports (CS) by an authorized administrator of ScholarWorks@UTEP. For more information, please contact Iweber@utep.edu. 


\section{Optimization of Quadratic Forms and t-norm Forms on Interval Domain and Computational Complexity}

\author{
Milan Hladík \\ Charles University \\ Faculty of Mathematics and Physics \\ Department of Applied Mathematics \\ Malostranské nám. 25 \\ 11800, Prague, Czech Republic \\ milan.hladik@matfyz.cz
}

\author{
Michal Černý \\ Department of Econometrics \\ University of Economics, Prague \\ W. CHurchill's sq. 4 \\ 13067 Prague, Czech Republic \\ cernym@vse.cz
}

\author{
Vladik Kreinovich \\ Department of Computer Science \\ University of Texas at El Paso \\ $500 \mathrm{~W}$. University \\ El Paso, Texas 79968, USA \\ vladik@utep.edu
}

\begin{abstract}
We consider the problem of maximization of a quadratic form over a box. We identify the NP-hardness boundary for sparse quadratic forms: the problem is polynomially solvable for $O(\log n)$ nonzero entries, but it is NP-hard if the number of nonzero entries is of the order $n^{\varepsilon}$ for an arbitrarily small $\varepsilon>0$. Then we inspect further polymonially solvable cases. We define a sunflower graph over the quadratic form and study efficiently solvable cases according to the shape of this graph (e.g. the case with small sunflower leaves or the case with a restricted number of negative entries). Finally, we define a generalized quadratic form, called t-norm form, where the quadratic terms are replaced by t-norms. We prove that the optimization problem remains NP-hard with an arbitrary Lipschitz continuous t-norm.
\end{abstract}

\section{INTRODUCTION}

In this paper we elaborate the problems outlined in [4] in more details. In that work we studied processing imprecise data from multiple sources which interact together. The interaction among inputs $x_{1}, \ldots, x_{n}$ is formalized by a function $f\left(x_{1}, \ldots, x_{n}\right)$ which cannot be written in a separable form as $\sum_{i=1}^{n} f_{i}\left(x_{i}\right)$ (for some functions $\left.f_{i}\right)$. An example is a quadratic form $x^{T} A x$ with nonzero off-diagonal entries, which is studied in this paper. Then we consider a more general form of pairwise interactions: formally, we replace the bilinear terms $x_{i} x_{j}(i \neq j)$ from $x^{T} A x$ by so-called $t$-norms (which can be regarded as generalizations of the "AND" logical connective).

The general question is: when the inputs $x_{1}, \ldots, x_{n}$ are imprecise but are known to be in given compact intervals

$$
\boldsymbol{x}_{1}=\left[\underline{x}_{1}, \bar{x}_{1}\right], \ldots, \boldsymbol{x}_{\boldsymbol{n}}=\left[\underline{x}_{n}, \bar{x}_{n}\right],
$$

and we are given a function $f: \mathbb{R}^{n} \rightarrow \mathbb{R}$, can we find tight bounds for $f\left(x_{1}, \ldots, x_{n}\right)$ ? Formally, denoting

$$
x=x_{1} \times \cdots \times x_{n},
$$

the problem reduces to the computation of $\sup _{x \in \mathbb{R}^{n}}\{f(x) \mid x \in \boldsymbol{x}\}$ and $\inf _{x \in \mathbb{R}^{n}}\{f(x) \mid x \in \boldsymbol{x}\}$. Here, the expression "to find the bounds" refers to computational complexity: We are to determine under which conditions the bounds can be evaluated in polynomial time and when the computation is NP-hard.
Recall that in general, finding the tight bounds for a general function $f$ need not be recursive. This is why various classes of functions of interest in data processing need to be studied separately.

In this text, bold symbols-such as $\boldsymbol{x}$-refer to $n$ dimensional intervals of the form (1). The real $n$-vectors of lower and upper bounds are denoted by $\underline{x}$ and $\bar{x}$, respectively, and we write $\boldsymbol{x}=[\underline{x}, \bar{x}]$ for short.

Basics in computations complexity and interval computing can be found e.g. in [6].

\section{QUADRATIC FORMS ON INTERVAL DOMAIN}

Consider a quadratic form $f: \mathbb{R}^{n} \rightarrow \mathbb{R}$

$$
\begin{aligned}
f(x) & =b^{T} x+x^{T} A x \\
& =\sum_{i=1}^{n} b_{i} x_{i}+\sum_{i, j=1}^{n} a_{i j} x_{i} x_{j}
\end{aligned}
$$

restricted to a given interval domain $\boldsymbol{x}=[\underline{x}, \bar{x}]$. It is known that computing the range of $f$ on $\boldsymbol{x}$, i.e.,

$$
\begin{aligned}
\underline{f} & :=\min f(x) \text { subject to } x \in \boldsymbol{x}, \\
\bar{f} & :=\max f(x) \text { subject to } x \in \boldsymbol{x}
\end{aligned}
$$

is an NP-hard problem. This is true even for $A$ positive definite, in which case computing $f$ is polynomial whereas computing $\bar{f}$ is NP-hard.

Assumption. For simplicity of exposition, we focus only on computation of $\bar{f}$ in the sequel. We will also assume for the remainder of the paper that $f(x)$ is convex (i.e., that $A$ is positive semidefinite).

\section{SPARSE QUADRATIC FORMS}

Suppose that the matrix $A$ is sparse, that is, most of the offdiagonal entries are zero. Becomes the problem of computing the range tractable?

In this section it is sufficient to fix

$$
\boldsymbol{x}=[0,1]^{n} .
$$


Proposition 1. The problem of computing $\bar{f}$ remains $N P$ hard even when the number of off-diagonal non-zeros in $A$ is bounded by $\mathcal{O}\left(n^{1 / k}\right)$.

Proof: Let $f(x)=b^{T} x+x^{T} A x$ be a quadratic function on $\mathbb{R}^{n}$. Consider the quadratic form

$$
g(x, y):=f(x)+\sum_{i=1}^{m}\left(2 y_{i}-1\right)^{2} .
$$

Then the maximum of $g(x, y)$ on $[0,1]^{n+m}$ is the same as the maximum of $f(x)$, shifted by the amount of $m$. That is,

$$
\bar{g}=\bar{f}+m .
$$

Putting $m:=n^{2 k}$ we get that the quadratic form $g(x, y)$ of dimension $d=n+m$ has $\mathcal{O}\left(d^{1 / k}\right)$, non-zero off-diagonal entries in the corresponding matrix. Since $f(x)$ was an arbitrary quadratic form, computing the range of $g(x, y)$ is NP-hard, too.

Corollary (to the proof). Under the assumption of Proposition 1 , it is NP-hard to approximate $\bar{f}$ with a given (arbitrarily large) absolute error. This follows from the fact that the maximum of a quadratic form is known to be NP-hard to approximate with an absolute error [1], and (2) does not change the absolute error.

On the other hand, approximating $\bar{f}$ with a relative error can be done efficiently via semidefinite ralaxation even for a nonconvex $f(x)$, see [5].

Proposition 2. The problem becomes polynomial if the number of off-diagonal non-zeros in $A$ is bounded by $\mathcal{O}(\log n)$.

Proof: Denote

$$
I:=\left\{i=1, \ldots, n \mid \exists j \neq i: a_{i j} \neq 0\right\} .
$$

Now, $f(x)$ can be expressed as

$$
f(x)=\sum_{i \notin I}\left(b_{i} x_{i}+a_{i i} x_{i}^{2}\right)+\sum_{i, j \in I} a_{i j} x_{i} x_{j},
$$

and its maximum as

$$
\bar{f}=\max _{x \in \boldsymbol{x}}\left(\sum_{i \notin I}\left(b_{i} x_{i}+a_{i i} x_{i}^{2}\right)\right)+\max _{x \in \boldsymbol{x}}\left(\sum_{i, j \in I} a_{i j} x_{i} x_{j}\right) .
$$

The first term in (3) is computed easily as

$$
\max _{x \in \boldsymbol{x}}\left(\sum_{i \notin I}\left(b_{i} x_{i}+a_{i i} x_{i}^{2}\right)\right)=\sum_{i \notin I} \max _{x_{i} \in \boldsymbol{x}_{i}}\left(b_{i} x_{i}+a_{i i} x_{i}^{2}\right),
$$

and maximizing a univariate quadratic function is a trivial task.

The second term in (3) requires maximizing a quadratic function on an interval domain in dimension $\mathcal{O}(\log n)$. Hence, by brute force, we find the maximum [2] in exponential time w.r.t. $\mathcal{O}(\log n)$, which is polynomial w.r.t. $n$.

\section{Polynomial CASES BASED ON SUNFLOWER GRAPHS}

Without loss of generality assume that $A$ is upper triangular and that $\boldsymbol{x}=[0,1]^{n}$. Consider the graph $G=(V, E)$, where $V=\left\{x_{1}, \ldots, x_{n}\right\}$ and $\left\{x_{i}, x_{j}\right\}$ is an edge of $G$ if and only if $a_{i j} \neq 0$. So we are in fact maximizing a quadratic form $f(x)$ on the graph $G$ (see Chapter 10 of [3]).

Let $D \subseteq V$ be a vertex cut such that the graph $G^{\prime}=$ $\left(V \backslash D, E^{\prime}\right)$ after removing the cut $D$ consists of connected components of vertex size $\mathcal{O}(\log n)$. Suppose further that the size of the cut is $|D|=\mathcal{O}(\log n)$. (A graph with such cut is sometimes called sunflower graph, see Figure 1.) Then the cut is associated with $|D|$ variables. Hence we can process all $0 / 1$-assignments of these variables. There are at most $2^{|D|}$ such assignments. For every such assignments, we resolve the problem by brute force in each of the components. Therefore, the overall time complexity is

$$
\begin{aligned}
2^{|D|} & \left(T_{1}+T_{2}+\cdots+T_{k}\right) \\
& \leq 2^{\mathcal{O}(\log n)}\left(2^{\mathcal{O}(\log n)}+2^{\mathcal{O}(\log n)}+\cdots+2^{\mathcal{O}(\log n)}\right) \\
& \leq \operatorname{poly}(n),
\end{aligned}
$$

where $T_{i}$ is time complexity of maximization over $i$ th component, $k \leq n$ is the number of components, and $\operatorname{poly}(n)$ is a polynomial in $n$.

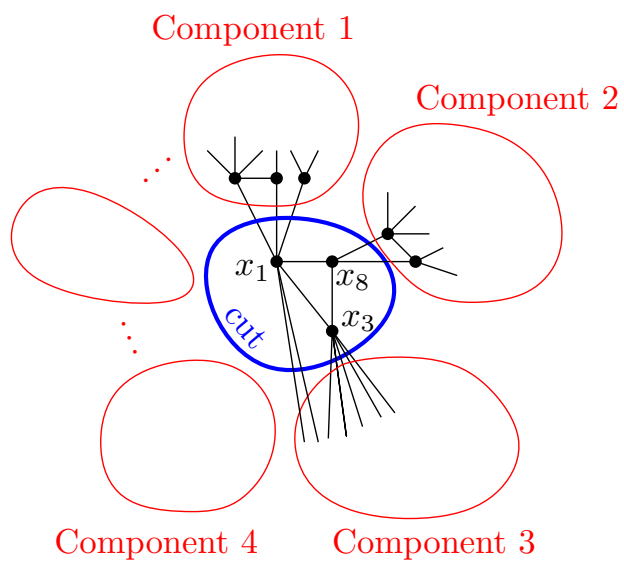

Fig. 1. A sunflower graph with a cut of $\operatorname{size} \mathcal{O}(\log n)$ and components of size $\mathcal{O}(\log n)$.

A problem. How to find a suitable cut? This is an open challenging question. Notice that minimum cut splitting graph $G$ into two components can be found efficiently by means of linear programming. Nevertheless, incorporating restrictions on size of the components seems a hard problem.

\section{Special graphs}

The above reasoning can be extended even to the components larger than $\mathcal{O}(\log n)$, but having a special structure. So, we will now discuss a few of special graphs possessing a suitable structure. For the sake of simplicity of exposition, we will illustrate it on the graph $G=(V, E)$. 
Few negative coefficients: Provided that all coefficients are nonnegative, that is, $b_{i} \geq 0$ and $a_{i j} \geq 0$ for all $i, j \in\{1, \ldots, n\}$, then the optimal solution is simply $x=$ $(1, \ldots, 1)^{T}$. If it is not the case, we can still effectively compute an optimal solution as long as the number of negative coefficients $b_{i}$ and $a_{i j}$ is small. Define a cut $D$ to contain all variables incident with negative coefficients:

$$
D:=\left\{x_{i} ; b_{i}<0 \text { or } a_{i j}<0 \text { for some } j\right\} .
$$

If $|D|=\mathcal{O}(\log n)$, then we are done since applying the cut we obtain a subproblem with nonnegative coefficients and the $0 / 1$-variables in $D$ can be tested brute-force in time $2^{|D|}=$ $\operatorname{poly}(n)$.

\section{Other special graphs}

Assume now for simplicity in the remainder of this section that the domain of variables is $\boldsymbol{x}=[-1,1]^{n}$. Further assume that $b_{i}=0$ for every $i$.

Trees: If $G$ is a tree, then maximizing the quadratic function on $G$ is easy: Take an arbitrary vertex in $x_{i} \in G$ as a root, and distinguish two assignments $x_{i}= \pm 1$. For each assignment, the remaining variables associated with $G$ have determined values. Sorting the vertices according to some tree search algorithm, we put $x_{j}:=\operatorname{sgn}\left(a_{i j} x_{i}\right)$ when $x_{i}$ precedes $x_{j}$.

Planar graphs: The above class can be extended to planar graphs with $\mathcal{O}(\log n)$ faces because by removing $\mathcal{O}(\log n)$ vertices we obtain a tree.

Bipartite graphs: Complete bipartite graphs $K_{m, n}$ and their subgraphs are also efficiently processed provided $a_{i j} \leq 0$ for $i \neq j$. The variables associated with the first set of vertices will be set as $x_{i}:=1$, and the others $x_{i}:=-1$.

If the assumption $a_{i j} \leq 0$ for $i \neq j$ is not satisfied, then the bipartite graph is still efficiently processed as long as $m=$ $\mathcal{O}(\log n)$, in which case the vertex cut $D$ is the smaller of those two subsets.

Remark. For related results see [7].

\section{T-NORM FORMS}

Recall that a t-norm is a function $T:[0,1]^{2} \rightarrow[0,1]$ satisfying:

- commutativity: $T(a, b)=T(b, a)$,

- monotonicity: $a \leq c, b \leq d \Rightarrow T(a, b) \leq T(c, d)$,

- associativity: $T(a,(T(b, c)))=T(T(a, b), c)$,

- 1 is identity element: $T(a, 1)=a$.

From the definition, we immediately have

$$
T(0,0)=T(0,1)=T(1,0)=0, \quad T(1,1)=1 .
$$

Given t-norms $T_{i j}$, the question is how easy is evaluation of the $t$-norm form

$$
f_{T}(x)=\sum_{i=1}^{n}\left(b_{i} x_{i}+a_{i i} x_{i}^{2}\right)+\sum_{i \neq j} a_{i j} T_{i j}\left(x_{i}, x_{j}\right)
$$

on a given interval domain $\boldsymbol{x}$.
Proposition 3. Maximizing a t-norm form on $\boldsymbol{x}=[0,1]^{n}$ is $N P$-hard even if we choose and fix for every $T_{i j}$ a Lipschitz continuous t-norm, that is,

$$
\left|T_{i j}(x)-T_{i j}\left(x^{\prime}\right)\right| \leq \alpha \cdot\left\|x-x^{\prime}\right\|,
$$

where $\alpha$ is a Lipschitz constant and $\|\cdot\|$ is any vector norm.

Proof: Let $f(x)=b^{T} x+x^{T} A x$ be a convex quadratic function on $\mathbb{R}^{n}$. Consider the t-norm form

$$
\begin{aligned}
f_{T}(x):= & b^{T} x+\sum_{i=1}^{n} a_{i i} x_{i}^{2}+\beta \sum_{i=1}^{n}\left(2 x_{i}-1\right)^{2} \\
& +\sum_{i \neq j} a_{i j} T_{i j}\left(x_{i}, x_{j}\right) .
\end{aligned}
$$

By the Lipschitz continuity assumption, for sufficiently large $\beta$ the function $f_{T}(x)$ is convex. Thus the maximum of $f_{T}(x)$ is attained in a vertex of $\boldsymbol{x}$. However, on a set of vertices $x \in\{0,1\}^{n}$,

$$
f_{T}(x)=f(x)+\beta n
$$

since $T_{i j}\left(x_{i}, x_{j}\right)=x_{i} x_{j}$ and $\left(2 x_{i}-1\right)^{2}=1$. This means that the maximum of $f_{T}(x)$ is the same as the maximum of $f(x)$, shifted by the amount of $\beta n$. Since maximizing $f(x)$ on $\boldsymbol{x}$ is NP-hard, maximizing t-norm forms on $\boldsymbol{x}$ is NP-hard, too.

Remark 1. It is interesting that the proof does not require all the axioms of a $t$-norm. Basically, we used (4) only. Thus the statement holds true for any Lipschitz continuous functions $T_{i j}$ satisfying (4).

Notice that the commonly used t-norms satisfy the assumption of the proposition:

- product t-norm $T(x, y)=x y$ (in this case, the t-norm form is a quadratic form),

- minimum t-norm $T(x, y)=\min \{x, y\}$,

- Łukasiewicz t-norm

$$
T(x, y)=\max \{0, x+y-1\},
$$

- nilpotent minimum t-norm

$$
T(x, y)= \begin{cases}\min \{x, y\} & \text { if } x+y>1, \\ 0 & \text { otherwise, }\end{cases}
$$

- Hamacher product t-norm

$$
T(x, y)= \begin{cases}0 & \text { if } x=y=0, \\ \frac{x y}{x+y-x y} & \text { otherwise. }\end{cases}
$$

On the other hand, the drastic t-norm defined as

$$
T(x, y)= \begin{cases}\min \{x, y\} & \text { if } \max \{x, y\}=1 \\ 0 & \text { otherwise }\end{cases}
$$

does not satisfy the assumption.

\section{ACKNOWLEDGMENTS}

M. Hladík was supported by the Czech Science Foundation Grant P403-18-04735S. M. Černý was supported by the Czech Science Foundation Grant P402/12/G097. V. Kreinovich was supported in part by the National Science Foundation Grant HRD-1242122 (Cyber-ShARE Center of Excellence). 


\section{REFERENCES}

[1] M. Černý $M$ and M. Hladík M. The complexity of computation and approximation of the $t$-ratio over one-dimensional interval data. Computational Statistics \& Data Analysis 80, 2014, 26-43.

[2] J.-A. Ferrez, K. Fukuda, and T. Liebling. Solving the fixed rank convex quadratic maximization in binary variables by a parallel zonotope construction algorithm. European Journal of Operational Research 166, 2005, 35-50.

[3] B. Gärtner and J. Matoušek. Approximation Algorithms and Semidefinite Programming. Springer, 2012.

[4] M. Hladík, M. Cerný, and V. Kreinovich, "When Is Data Processing under Interval and Fuzzy Uncertainty Feasible: What If Few Inputs Interact? Does Feasibility Depend on How We Describe Interaction?" These Proceedings.

[5] Nesterov Yu. Semidefinite relaxation and nonconvex quadratic optimization. Optimization Methods \& Software 9 (1-3), 1998, 141-160.

[6] V. Kreinovich, A. Lakayev, J. Rohn, and P. Kahl, Computational Complexity and Feasibility of Data Processing and Interval Computations, Kluwer, Dordrecht, 1998.

[7] Yu G.-D. (2014). Quadratic forms on graphs with applications to minimizing the least eigenvalue of signless Laplacian over bicyclic graphs. Electronic Journal of Linear Algebra 27, article 13. 